\title{
Perception of interpolated position and orientation by vision and active touch
}

\author{
SUSAN J. LEDERMAN ${ }^{1}$ AND M. M. TAYLOR ${ }^{2}$ \\ DEFENCE RESEARCH ESTABLISHMENT, TORONTO
}

Vision and active touch lead to similar patterns of constant error for the perception of interpolated position in twodimensional and one-dimensional regions, though the errors for touch are larger than those for vision. The error patterns for the orientation of a radius of a semicircle are more complex, but can be interpreted as due to the interaction of two sets of anchors rather than the single pair available for the linear interpolation. The greater size of the touch errors is interpreted as due to a relative overestimation of larger distances by active touch or of smaller distances by vision.

Numerous studies of visual interpolation have been reported, especially in the context of meter reading (e.g., Bartlett, Reed, \& Duvoisin, 1949; Carr \& Garner, 1952; Chapanis \& Leyzorek, 1950). Typically, the results have been that the errors that occur are towards the ends of the scale, except possibly for stimuli very near the end of the interpolation interval.

Henry $(1893,1895)$ and Pillsbury (1894) found a similar tendency in the localization of touches on the arm and hand, for which errors were typically towards such anchor points as the wrist, elbow, and knuckles. In a heroic experiment, Boring (1915) found analogous errors for the distance inside the esophagus from the throat to the stomach, where electric shocks were typically located much too near to the throat or the stomach.

It does not appear that the interpolation effect has been studied for active touch. In the experiments reported here, we have examined visual and tactile interpolation in two dimensions of a dot or bump on a rectangular card, the unidimensional tactile interpolation of a bump on a thin rod, and the visual and tactile perception of the orientation of a radius of a semicircle. This latter may be considered an interpolation in the angular interval from 0 deg to $180 \mathrm{deg}$.

In some ways, the visual part of the orientation experiment replicates part of a study by Jastrow (1893), which indicated that angle estimation was subject to a pattern of errors similar to that later found for linear interpolations. The visual part of the experiment involving dots on cards was a replication of part of a study on anchoring (Taylor, 1961) and the brass rod experiment was a hitherto unpublished preliminary study for that same paper.

Both Gibson (1962) and more particularly Katori and Natori (1967) have pointed out that tactile perception is akin to visual perception but only if the touching is active. Further, Katori and Natori have shown that if the touching is restricted appreciably, the method an observer uses to redraw a figure perceived visually differs from the method he uses to reproduce even an actively touched figure. If touching is free, and particularly if it is two-handed, the observer tends to use the same drawing technique for reproducing felt and seen patterns. In the experiments reported here, the observers were free to use any touching method they liked, provided it did not involve the use of explicit measuring devices such as fingertip spread. If Gibson and Katori and Natori are correct, we should expect the results for touch to be similar to the results for vision.

\section{EXPERIMENTS}

Experiment 1. Dots Placed on Cards

Subjects. The eight Ss were selected for handedness, half being right-handed for writing, half left-handed. There were four housewives, three men approximately 20 years of age serving in the Canadian Armed Forces, and one male member of the professional staff of DRET.

Stimulus materials. The stimuli consisted of $3 \times 5 \mathrm{in}$. high-quality index cards that were ruled on one side. Owing to the accuracy of measurement required, we attempted to ensure that all cards were exactly of nominal size. Most commercial cards are not cut to the required tolerance; however, we managed to obtain cards well within $1 \mathrm{~mm}$ of the size desired. A circular indentation, about $0.8 \mathrm{~mm}$ in diam, was made with a ball-tipped stylus on the ruled side of each index card. The bulge thus formed on the plain side was marked with a green felt pen. There were 64 cards, each with one such stimulus dot. Dots were placed to form a symmetrical pattern overall, identical to that used by Taylor (1961) (Fig. 1). The complete pattern was marked on a single card, called the master card. Each of the 64 cards had a code number on the back to identify the position of the stimulus on the master card.

Experimental procedure. Subjects were tested individually. The $S$ was seated at one end of a desk, which was covered with black plastic. A black curtain hung down to the desk top, about 8 in. in front of the $\mathrm{S}$. The $\mathrm{E}$ was seated at one side of the table so that she could work behind the curtain without being observed by the $S$, but could watch him in a mirror placed above and behind him.

When $S$ was locating the position of the dot by touch, one complete presentation cycle was as follows. $S$ had a stack of unmarked response cards to his left, the ruled surface facing upwards. He placed a card in front of him within a small area marked on the table, so that the long edges roughly paralleled the desk edge. E called out the code number of a particular stimulus card and $S$ wrote it in the top right-hand corner of the ruled surface. $S$ then turned the response card over and placed his hands underneath the curtain. In the meantime, $\mathrm{E}$ had placed the stimulus card within easy reach. There was no way in which $S$ could see the stimulus card, and no limit was placed on the length of time allowed for examination. When $S$ was satisfied that he knew the position of the dot, he withdrew his hands and placed a small mark with a sharpened lead pencil in the appropriate place on the blank response card. Following the procedure used by Taylor (1961), S was allowed to feel the stimulus card again if necessary. When he was finished, $S$ placed the marked response card under the curtain for $\mathrm{E}$ to take, and was ready to begin a new cycle.

The vision cycle was similar to the procedure used for touch. While $S$ wrote the code number on the back of the response card, E placed a stimulus card on the $S$ 's end of a long narrow cardboard tray covered with black plastic. When $\mathbf{S}$ was ready, he pulled the tray from behind the curtain so as to cover the response card completely and bring the stimulus card into view. S was instructed to pull the tray perpendicular to the table edge by 


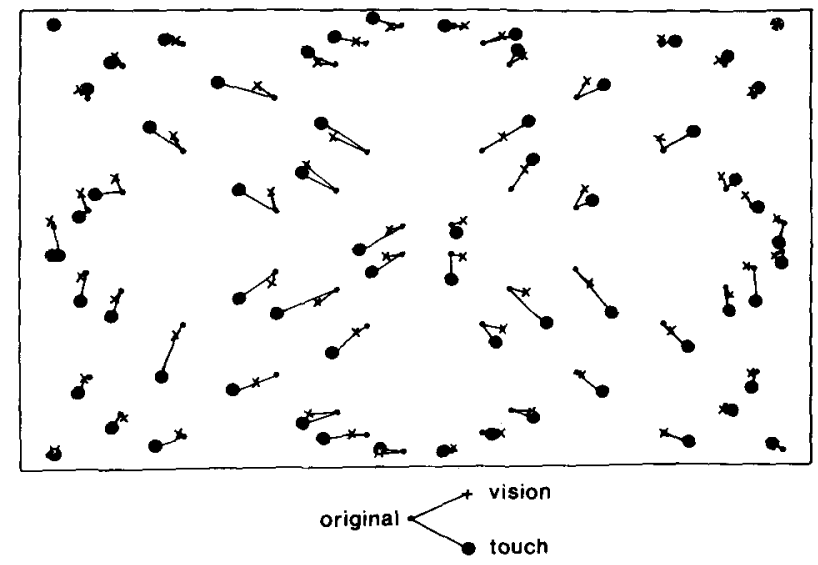

Fig. 1. The overall pattern of stimulus dots used in the first experiment is shown by the small dots. The average vision response is shown by the cross associated with each stimulus, and the average touch response by the large filled circle.

guiding it along an appropriately placed piece of cardboard. The card lay on the tray during S's visual examination. When he was finished, $S$ pushed the tray back under the curtain, and made his response as described above.

After each pass through the stimulus pack the cards were reshuffled. One pass, using either touch or vision, constituted an experimental session.

Experimental design. Each $\mathrm{S}$ was required to reproduce both felt and seen dots. Half of the Ss began with three vision sessions, followed by three touch sessions, while the rest began with touch and concluded with vision. Only one session was run per day. Originally, Ss were grouped for analysis according to sex and handedness. Since the data showed no trends for either effect that would reliably distinguish the various groups, for purposes of further analysis all Ss were treated as members of the same population.

We initially thought that performance might improve from day to day with practice. The first few Ss did their first three sessions on consecutive days and their second three on further consecutive days after an intervening weekend. There was no evidence of

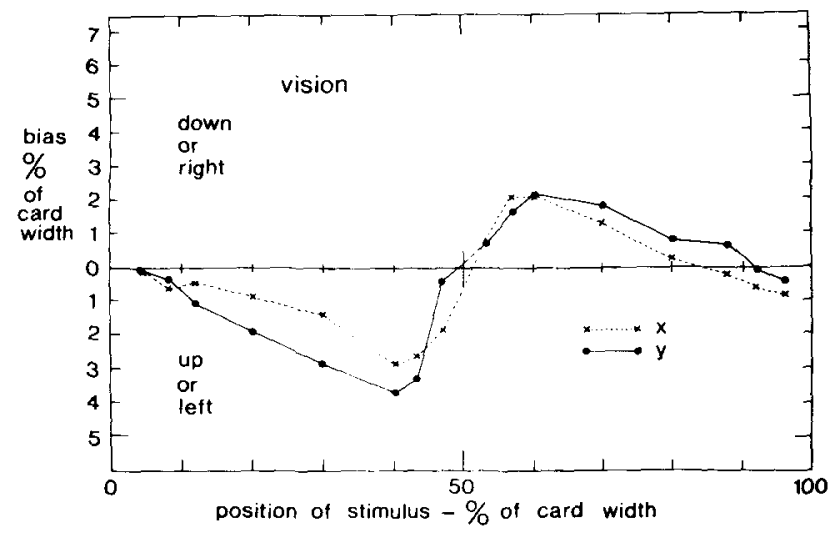

Fig. 2. Vision errors. Average reproduction error in $\mathrm{X}$ as a function of the $X$ position of the stimulus, and in $Y$ as a function of the $Y$ position of the stimulus. The unit of measurement is $\%$ of the dimension being plotted. Since the width of the card was 5 in., the unit of $X$ is .05 in. Similarly, the unit in $\mathrm{Y}$ is .03 in.

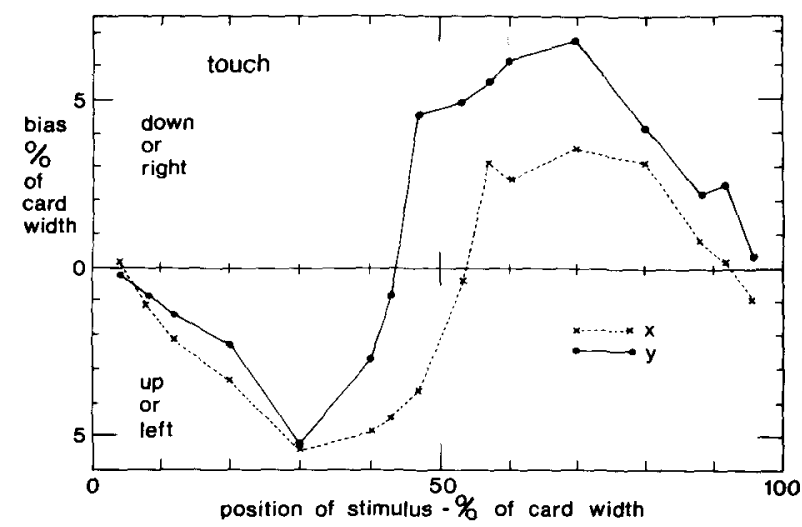

Fig. 3. Touch errors. Scales same as Fig. 2.

learning, so later Ss were run as convenient.

Results. The results from the dot experiment are presented in Figs. 14. Figure 1 shows the overall pattern of the stimulus dots as well as the average biases in reproduction of the dots when perceived visually and through active touch. The mean of the errors across days was determined for each $S$, and the spatial median of the eight resulting scores was found by the method of convex hulls (Taylor, 1961), providing the overall average reproduction error for each stimulus dot. As may be seen, both vision and touch biases tend toward the corners of the card; the major apparent difference between the two is in magnitude, the touch errors being consistently larger.

In agreement with the finding of Taylor (1961), the error in $X$ did not depend on the $Y$ position of the dot, and vice versa; this permitted us to collapse $X$ scores across $Y$ and $Y$ scores across $X$, respectively. Figures 2,3 , and 4 show the constant errors of reproduction, as a function of dot position, in the $X$ and $Y$ dimensions for vision, touch, and "touch minus vision." The position of the stimulus dots is described in terms of percentage of the card size in $\mathrm{X}$ and $\mathrm{Y}$ dimensions, the origin being taken as the top left corner of the card, as in reading a book. Thus, positive bias means error upwards or to the left; negative bias refers to error downward or to the right of the stimulus dot. Each point in Figs. $2-4$ is based upon 24 reproductions of each of four dots.

Visual biases are shown in Fig. 2. It can be seen that the absolute errors in both $X$ and $Y$ are smallest at the edges and center of the card. Outward errors predominate. Stimulus dots on

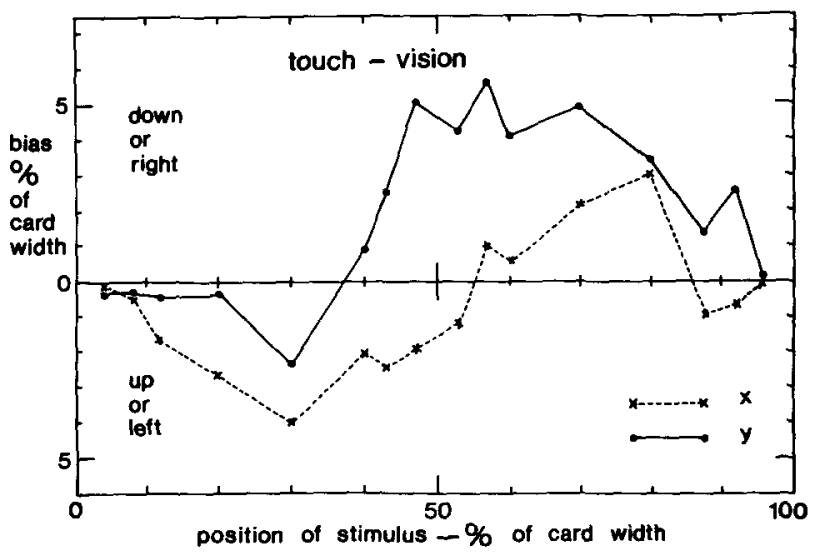

Fig. 4. Difference between touch and vision errors. Scales same as Fig. 2. 
the left side of the card are reproduced further to the left, and stimuli on the right further to the right, except for the few points on the extreme right. Dots located in the top half of the card are generally reproduced closer to the top while those in the bottom half are moved even lower down. Touch errors (Fig. 3) show the same pattern but are considerably larger than those for vision.

Although in one section of the experiment Ss perceived the stimulus dots through active touch, they were nevertheless required to look at the response card in order to reproduce these dots. We assumed that the components of error arising in the response per se would be the same regardless of the mode of perception. Since vision may be considered to be the ordinary manner of perceiving things spatial, the vision error was subtracted from the overall touch errors to obtain "pure" touch perception effects. Figure 4 represents the results of this process. The differences between errors with touch perception and visual perception show the same pattern as the visual perceptionresponse errors, but are larger.

Inversions. Seven of the touch responses and one visual response were far removed from the main cluster of responses made by all Ss to those stimuli. In all these cases, a mirror-inversion of the faulty response about the $\mathrm{X}$ axis brought the point well into the ordinary range of responses. We assume that Ss actually coded their percept in a form that permitted separate errors in the distances from the card edges and in the left-right sign of the position. These suspected inversions were ignored in the main analysis of the data. A few other responses might have been inversions, but they were not as clear as those mentioned, and were included in the analyses without modification. Similar effects occurred in the experiments reported by Taylor (1961) but were not mentioned in the published paper.

\section{Experiment 2. Tactile Interpolation Along a Rod}

The data reported in this section were obtained in 1958 as part of the preliminary work for the studies reported in Taylor (1961). The stimulus materials were polished brass rods, $12 \frac{1}{2} \mathrm{~cm}$ long and approximately $1 \mathrm{~mm}$ in diam. On each rod was a blob of solder formed around a fine wire wrapped on the rod. The blob approximated a sphere $2 \mathrm{~mm}$ in diam. S put his hands through a hole in a 2-ft-square cardboard box, and was given a rod to feel. He could feel in any way he chose including turning the rod end for end, except that he was not permitted to make a direct estimate of the position of the blob by fingertip separation. When he was satisfied as to the position of the blob on the rod, he made his response by selecting one of a series of drawings on $3 \times 5$ in. index cards. Each drawing showed a 3-in. line with a small mark on it. There were 30 such drawings, with marks at positions evenly spaced from one end to somewhat beyond the middle of the drawn line. The tactile discrimination was never good enough to permit the $\mathrm{S}$ to select a response card unambiguously, so that the set of response cards may be considered as if it were a response continuum.

Sixteen undergraduate students in psychology at The Johns Hopkins University served as Ss. Each made two judgments of each of seven rods. The overall average error is shown as a function of the true position of the solder blob in Fig. 5. This average picture is reasonably representative of the results for individual Ss, although for blobs near the end of the rod, some Ss showed relatively greater or lesser reversal of the normal outward error tendency. The maximum average error in matching of tactile and visual interpolations was of the order of $4 \%$ of the card width. The tactile judgment was typically outward of the visual judgment, except for the usual end effect.

Both this study and the dot position study suggest that active touch is subject to the same interpolation errors as is vision, but

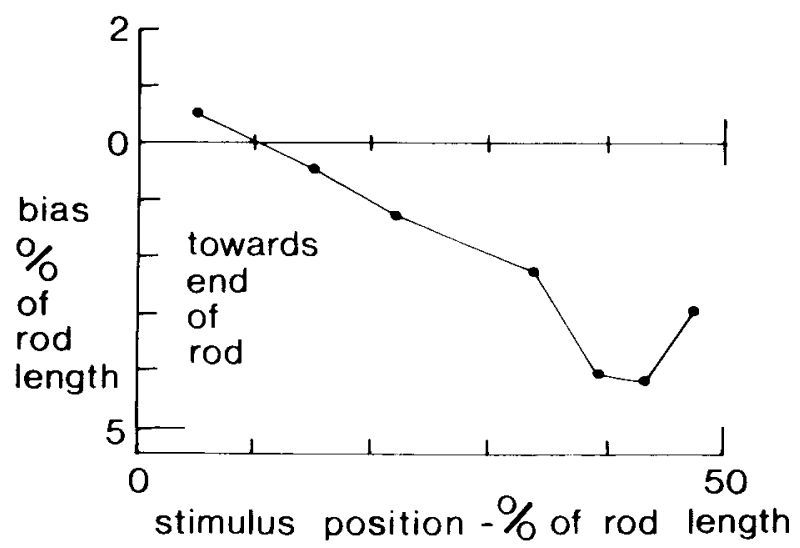

Fig. 5. Mean error in selecting a visual interpolation that corresponds to the felt position of a blob on a brass rod. The unit is \% of total length of interpolated interval, i.e., the length of the rod.

that the magnitude of the error in touch is about double that in vision.

\section{Experiment 3. Orientation of the Radius of a Semicircle}

Subjects. The Ss were the two authors, four Canadian Armed Forces men, and four students.

Apparatus. The apparatus is shown in Fig. 6. S viewed and controlled the rotation of a 6-in. white cardboard disk with a diameter drawn across it. The lower half of the disk was obscured by white cardboard with a black line drawn along the top edge, so that the disk looked like a semicircle with a radius drawn in a manipulable orientation. The surface behind the disk was also

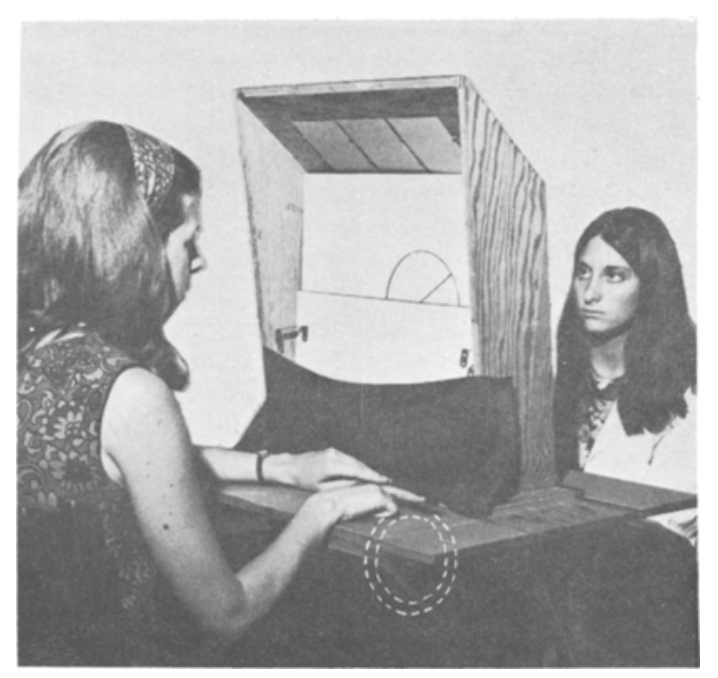

Fig. 6. The experimental apparatus for the orientation experiment in use. $S$ is holding a stimulus card face down in the left hand, while manipulating the control wheel (dotted white) with the right. The senior author is watching the protractor on the back of the apparatus preparatory to recording the response orientation. The edges of the visible semicircle of the response disk have been artificially enhanced in this reproduction. 
white cardboard. To rotate the disk, $\mathrm{S}$ moved with his fingertips a wheel (indicated with dashed lines in the figure) whose rubber tire just protruded above the flat surface of the table. Fingertip control of this type eliminates possible kinaesthetic cues to the orientation of the wheel and hence of the disk. A protractor on the same shaft as the disk permitted E, sitting at the back of the apparatus, to read the orientation of the line to the nearest $0.1 \mathrm{deg}$, which is much more accurate than were S's adjustments.

A curtain covered an aperture below the face of the apparatus. Ss could place their hands through the curtain to feel a stimulus card without ever actually seeing it.

Stimulus materials. Semicircles, about $61 / 2 \mathrm{~cm}$ radius, were cut from heavy white construction paper. Using a wheel pen to make lines in the form of a sequence of tiny raised bumps on the cards, radii were drawn to form two sets of angles, ranging from $5 \mathrm{deg}$ to $175 \mathrm{deg}$ in 5-deg steps, measured as opening from the right-hand edge of the semicircle. In addition, each set contained angles of $2 \mathrm{deg}, 88 \mathrm{deg}, 92 \mathrm{deg}, 178 \mathrm{deg}$, and a duplicate $90 \mathrm{deg}$. The two sets, therefore, consisted of 40 cards each. The lines were marked on the raised side with a fine brightly colored felt pen, one set brown, the other green. The techniques used to make these angles allowed the $S$ to see and feel the same stimulus cards.

Procedure. The $\mathrm{S}$ was seated in front of the apparatus, the $\mathrm{E}$ at the back. An experimental session was as follows: $S$ estimated one or the other set of angles beginning either with vision or with touch. Half the Ss began a session with vision and ended with touch, while the other half started with touch and ended with vision.

If an $\mathrm{S}$ was examining the cards visually, he held out his hand to the side of the apparatus to indicate that he was ready for a card, which $\mathrm{E}$ then gave him. S examined it until he was ready to estimate the size of the angle. He then turned the card so that he could no longer see it, and ajusted the wheel until the angle formed by the horizontal and the radius on the disk appeared to be the same as the angle he had just examined visually. He was allowed to re-examine the stimulus and re-adjust the wheel as long as he did not move the wheel while the stimulus was visible to him. When S completed the set of cards he was given a break if he wished, and then the second half of the session began.

To examine a stimulus card tactually $S$ placed both hands through the curtain below the face plate to where $E$ had placed the card. Any method of touching the card was allowed, and S was permitted to feel the angle as long and often as he liked, provided he did not have a hand on the card at the same time as he was moving the wheel. The same deck of cards was used for both visual and tactual examination in a given session but the two decks were alternated across Ss and from day to day for each S. Each $S$ was run for one session a day on three separate days. The order of presenting vision and touch was reversed from session to session for each $\mathrm{S}$.

Results. The results of the angle experiment are shown in Figs 7-13. Figure 7 shows the response error in degrees as a function of seen line orientation, Fig. 8 the same for felt lines, and Fig. 9 for the difference between the seen line and the same line felt in the same session. In each graph, the cross-hatched area represents the region within one standard error of the mean, indicating the reliability of the measures.

For linear interpolations, such as in Experiments 1 and 2, the main error tendency is towards the ends of the interpolation interval. Figure 7 shows that this tendency holds for the visually perceived lines, but only to a small extent over most of the range, and that the maximum deviation occurs near the ends rather than near the middle of the range. Figure 8 and the derived Fig. 9 show that the touch pattern is different, in that a pattern like the standard is repeated twice, once in the interval between $0 \mathrm{deg}$ and $90 \mathrm{deg}$ and once in the interval between $0 \mathrm{deg}$ and $180 \mathrm{deg}$. The touch errors are also much larger than the vision errors.

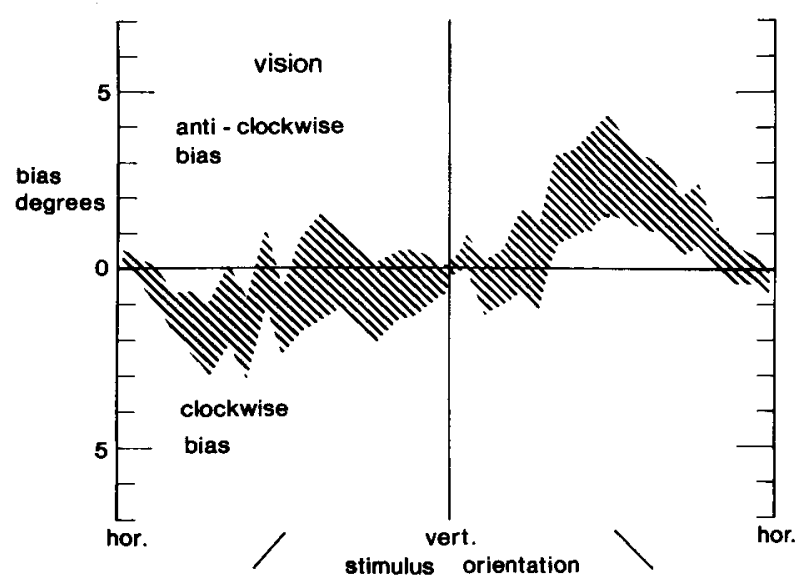

Fig. 7. The average response error for seen orientation. The crosshatched area represents the region within one standard error of the mean.

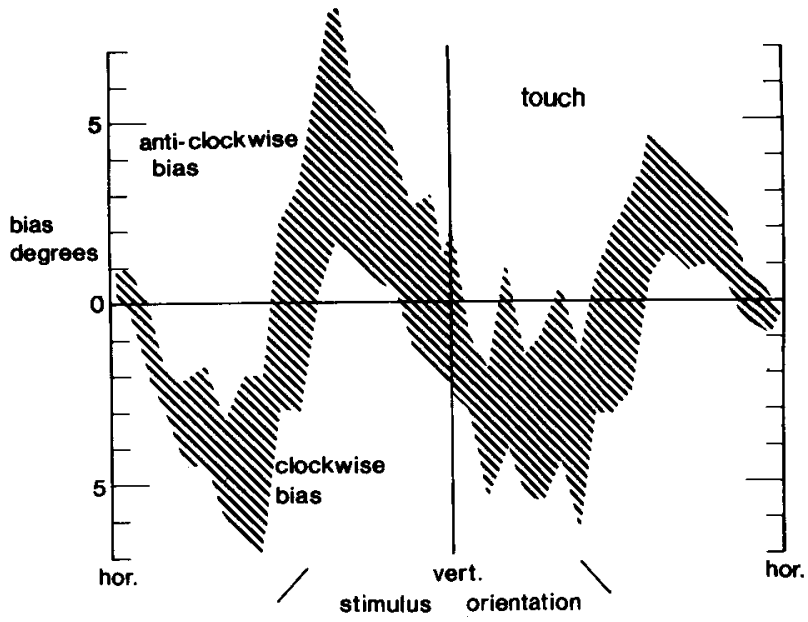

Fig. 8. Same as Fig. 7, but for touch errors.

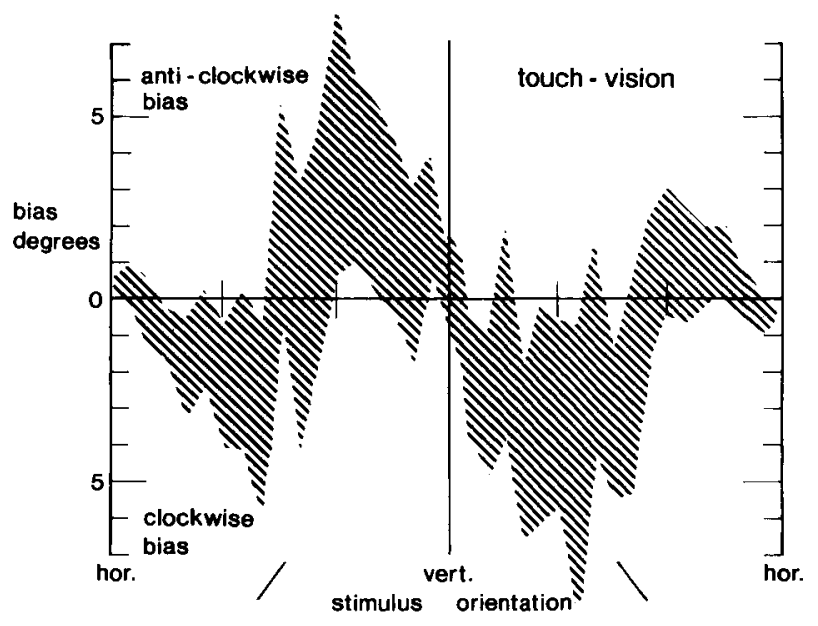

Fig. 9. Same as Fig. 7, but for the difference between touch and vision errors. 


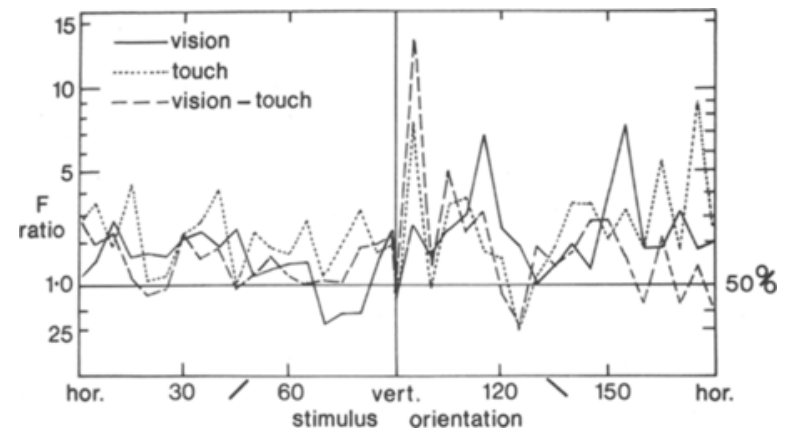

Fig. 10. F scores for between- vs within-S variances at each orientation for vision, touch, and difference errors. The ordinate scale is square-root. The general impression is that $F$ scores range around 2 rather than 1 , indicating that there is a definite but slight difference between-Ss in their response patterns.

Figures 7.9 are derived by averaging the data from all Ss. This procedure is statistically defensible, as is shown by Fig. 10. Each point in Fig. 10 is the score from a one-way analysis of variance, using mean square error between and within Ss in a particular condition as the numerator and denominator, respectively, of the $F$ ratio. Whichever stimulus orientation is considered, whether in vision, touch, or touch minus vision, the $F$ scores generally range around 2. Clearly, Ss do differ in their responses, but equally clearly the differences are slight, and not such as to be noticed within the precision of the experiment. Inspection of the data shows, furthermore, that much of the variation between Ss is due to changes in magnitude of the error pattern and not to gross changes in its shape.

The pattern of standard deviations of reproductions, derived from the mean squares within $\mathrm{Ss}$ of the analyses of variance, is shown for the vision, touch, and difference measures in Fig. 11. Note that in spite of the doubled pattern of constant error (Figs. 8 and 9) for the touch data, the standard deviations pattern has the same characteristic shape for all measures. The errors are low near the ends and middle of the 180-deg range, and high near the 45-deg and 135-deg angles. The standard deviation curves are, in fact, remarkably like those found by Taylor (1961) for linear interpolation. Note that the standard errors for touch, like the constant errors, are about twice those for vision. The measurement technique precludes our obtaining similar curves from the dot experiment of the present study for further comparison.

The curves of mean error (Fig. 8) suggested the possibility that the near-zero mean error in touch for orientations near $45 \mathrm{deg}$ and $135 \mathrm{deg}$ could have been artifacts due to the combination of some very high responses with some very low responses, few responses being accurate. Such a bimodal response distribution would, in fact, correspond with the authors' subjective impression of how angles in that range feel. We tested the response distributions for bimodality in the following way: If responses are all either high or low, then the errors of the three responses given to one stimulus angle by one $\mathrm{S}$ must be distributed either one on one side and two on the other side of zero, or else all three on the same side of zero. In the first case, he would show a small mean error and a large standard deviation, whereas in the second, a large mean error would go with a small standard deviation. Hence a correlation between mean error (absolute magnitude) and standard error across $S$ s should be negative for any angle giving a bimodal response distribution. On the other hand, if the response distribution were unimodal, large mean errors should occur more frequently when the standard deviation was large, and the tendency (obscured by other factors) should be for the correlation to be positive between mean error and standard deviation. The correlations in fact proved to be distributed around +.1 , very slightly positive, and certainly not supporting the hypothesis of a bimodal response distribution. No trend in the correlations across orientation was apparent, and in particular there was no tendency for the correlations to be negative for orientations near $45 \mathrm{deg}$ or $135 \mathrm{deg}$.

\section{DISCUSSION}

A major generalization from the three experiments is that, in both touch and vision, response estimates tend to be nearer the ends of the scale than are the corresponding stimuli. For placement of dots on a card, the pattern of constant errors for felt stimuli is very similar to that for the seen stimuli, except that the touch errors are larger. This same pattern is evident in the data from the brass rod experiment, in which the touch percept yields points closer to the ends of the scale than does the visual.

The comparison of visual and tactual perception is more complex in the case of the angle experiment. The graph of tactual errors clearly shows the standard pattern repeated twice, once between $0 \mathrm{deg}$ and $90 \mathrm{deg}$, and once between $90 \mathrm{deg}$ and $180 \mathrm{deg}$. The implication of this pattern is that the anchors for the touch scale are at the nearest flat edge of the semicircle and at $90 \mathrm{deg}$. The manner in which the Ss felt the card is consistent with this interpretation. With stimulus angles of appreciable size, the typical $\mathrm{S}$ would try to make a right angle by moving his fingers directly away from the card edge, and would then judge the angle with reference to the nearer edge and the derived right angle. This was by no means the only method of feeling used, but it occurred sufficiently often to warrant the statement that a derived 90 -deg orientation could serve as an anchor for the judgment of oblioue orientations.

The data for visually perceived angles are more complex. The graph (Fig. 7) shows the endward error pattern typical of the linear interpolation experiments, but with a considerably different form. In the linear interpolation experiments, the maximum deviation occurs very close to the midpoint of the interval, but in the visual angle experiment the maxima are rather nearer the endpoints. Furthermore, in the angle experiment, the central region of the interval shows very little constant error at all, despite the fact that the standard deviation has the standard pattern (Fig. 11).

The data for the visually perceived angles are consistent with the notion that the $S$ implicitly interpolates within two intervals

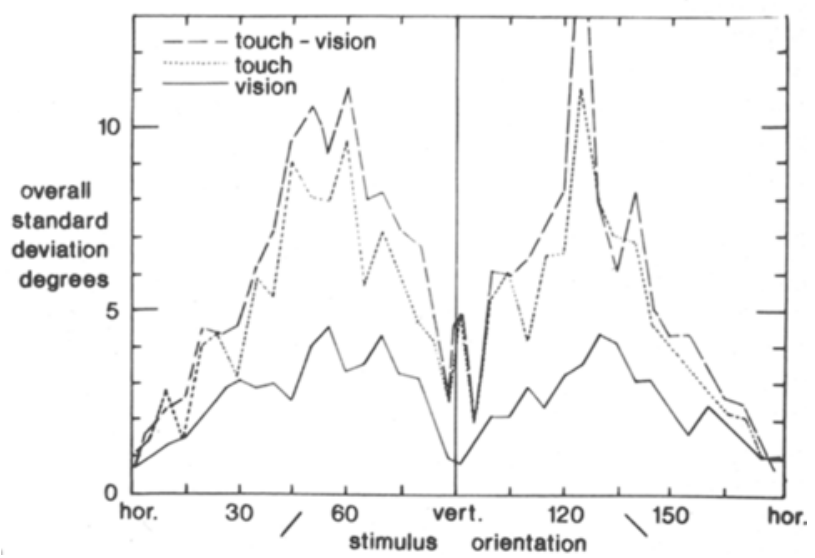

Fig. 11. Standard deviation of reproduced orientation as a function of stimulus orientation, for vision, touch, and the difference between touch and vision in the same session. 


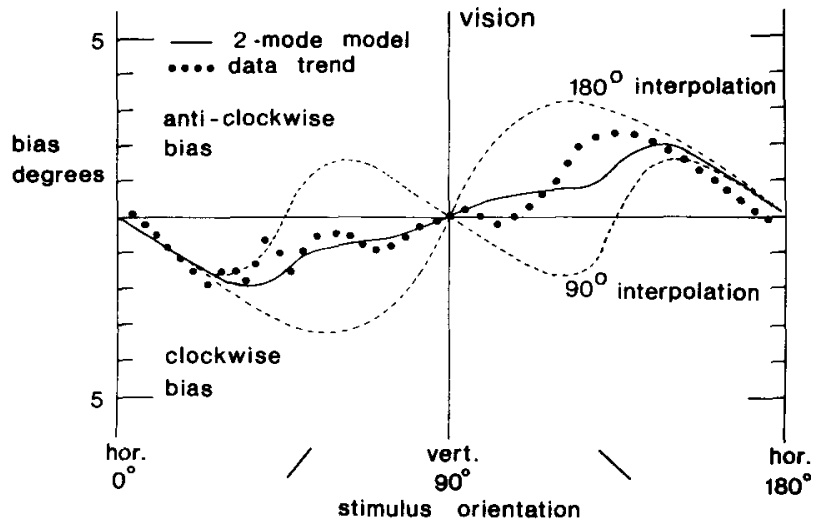

Fig. 12. The pattern of vision errors from Fig. 8 interpreted as the average of two independent error patterns, one based on a $180 \mathrm{deg}$ interval for interpolation and the other on a $90 \mathrm{deg}$ interval between either edge and the perpendicular. The dotted lines represent the hypothetical individual interpolation errors, the solid line the average. The solid line thus represents the predicted overt errors. The line of large dots represents the data trend from Fig. 8, not individual data points.

at once: one interval from $90 \mathrm{deg}$ to the nearest flat edge of the card, the other ranging over the whole $180 \mathrm{deg}$ (Fig. 12). Within each of these intervals, the standard error pattern of edgeward errors occurs covertly. Near the edges of the 180-deg interval, the two covert patterns add, but near the midpoint the 90-deg pattern gives errors toward $90 \mathrm{deg}$, whereas the 180 -deg pattern continues to give errors towards $0 \mathrm{deg}$ or $180 \mathrm{deg}$. Hence the two error tendencies oppose near $90 \mathrm{deg}$, yielding small overt errors.

The asymmetry in the visual angle data may possibly be accounted for by a slight asymmetry in the apparatus. The center of the disk was somewhat to the right of the center of the face plate, so that the disk edges were $4 \mathrm{in}$. and $8 \mathrm{in}$. from the right and left edges of the device, respectively. The apparatus originally was made for another experiment and adapted for this one. The asymmetry was not then considered important, but it is a complicating factor that prohibits discussion of the asymmetry in the data as being clearly an orientation effect.

If we accept the three propositions that the linear interpolation data represent some "standard" tendency for edgeward errors, that the touch data for angles demonstrate this same tendency when $90 \mathrm{deg}$ is used as an anchor, and that the vision data for angles represent a combination of effects due to simultaneous anchoring within a 90-deg sector and within a 180-deg sector, we are then left with two major questions: How does the standard pattern arise? Why are the touch errors ordinarily about double the vision errors?

The reproduction of seen dots on cards replicated, with similar results, the first experiment of Taylor (1961). The theory proposed in that paper may be used to describe the data of the present experiment. In brief, Taylor's 1961 theory proposed that the perceived distance from an "anchor point" of some unmarked "place" in a continuum was a nonlinear function of the physical distance, such as is shown in Fig. 13. This nonlinear function was not directly observable, since the act of marking the "place" provided another anchor point that itself distorted the perceived distance relationships. Although the function was not directly observable, changes in the function due to the addition of a stimulus point within an interpolation interval could be determined; a reproduction of the stimulus point onto a blank card provided a measure of the "place" on the blank card corresponding to the position of the stimulus point on the marked card. The reproduction error was the physical value of the amount by which the triply anchored (two card edges and the stimulus dot) stimulus function differed from that of the doubly anchored (two card edges only) blank card function at the perceived position of the stimulus dot. The triply anchored stimulus function and the doubly anchored response function are shown in Fig. 14. The theory is described in more detail in the original reference.

Consider now the differences between the tactual and the visual interpolations. The fact that the tactual displacements are larger than the visual is consistent with results from figural aftereffect studies. Taylor (1962) fitted a theoretical equation to the distance paradox data from a number of experiments in auditory, visual, and tactual spaces, and found that the scale parameter of the equation was the same for all the experiments except the single one on tactual width (Charles \& Duncan, 1959) for this experiment the scale parameter was about two and a half times larger than that found for the various visual and auditory spaces.

In Fig. 13, the perceived proportion of the interval that lies between the left edge and the marked point is dependent on the actual function relating perceived and physical distance. The more quickly the perception of large distances grows relative to that of small distances, the smaller the relative perceived distance to the nearer edge of the interpolation interval. Hence, if we assume that the tactual distance percept grows relatively more swiftly than does the visual as a function of physical distance, then the touched point must seem nearer the card edge. This is the consistent difference between touch and vision found in all three experiments. Note that this explanation of the relative sizes of the vision and touch displacements in no way relies on the correctness of the Taylor (1961) theory of the effect.

We must assume, then, that distance perceived tactually increases faster as a function of physical distance than does distance perceived visually. In the sense of S. S. Stevens (e.g., Stevens \& Guirao, 1963), we assume that the exponent of the psychophysical function is larger for touch distance than for visual distance. Cheng (1968) has reported results by Harris and Davidon bearing on this assumption. The touch exponent ranges from 1.1 to 1.3 whereas the visual is ordinarily (Stevens \& Guirao, 1963; Wong, 1963) around 1.0. The value quoted by Cheng for Harris's vision experiment is $\mathbf{1 . 0 5}$. In the light of these results, the value for vision of 1.5 quoted by Cheng for an experiment by Davidon seems anomalously high. On balance, it

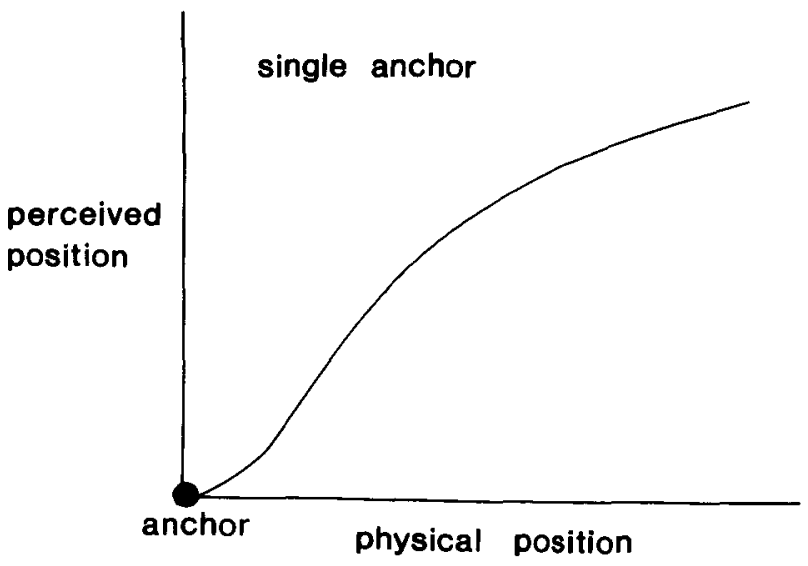

Fig. 13. A hypothetical function relating perceived distance from a single anchoring point to the physical distance from the anchor to some unmarked place. 


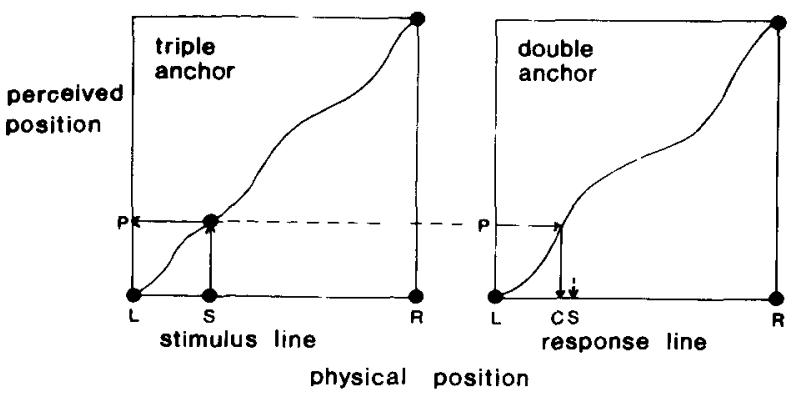

Fig. 14. The hypothetical function of Fig. 13 predicts the pattern of response errors. In the left-hand panel, the three anchors $L$ (left edge), $R$ (right edge) and $S$ (stimulus) combine to give a particular functional relationship between physical and perceived distances across the card, and hence to give a perceived position ( $P$ ) to the stimulus dot. In the right-hand panel, the blank response card has only two anchors, $L$ and $R$. These produce a different functional relationship between perceived and physical distances across the card, so that the perceived position of the stimulus, $P$, corresponds now with a different physical position, $C$. $C$ is the place at which the subject makes his response.

seems that the exponent for felt length is greater than that for seen length. Further indirect support comes from experiments by Churchill $(1959,1960)$ on visual and tactual interpolation of perceived width, in which tactual interpolated widths were consistently smaller than the visual interpolations between the same two standards. Churchill's data suggest that as stimulus width increases, the tactual perceived width increases at first more slowly and later more rapidly than the visual.

\section{SUMMARY}

In three experiments on interpolation, active touch has been shown to give results similar to those found for vision. There are differences, however, between the modalities. In the interpolation of angles, touch shows a very marked pattern of error towards $0 \mathrm{deg}$ (or $180 \mathrm{deg}$ ) and $90 \mathrm{deg}$, whereas vision gives rather smaller errors, mainly towards $0 \mathrm{deg}$ and $180 \mathrm{deg}$. This difference can be interpreted as due to the fact that in feeling for the angles, Ss pay greater attention to the 90-deg direction and to the nearer edge of the semicircle, thus effectively reducing the interpolation interval from $180 \mathrm{deg}$ to $90 \mathrm{deg}$. In vision, the two edges of the semicircle and the imagined 90-deg direction can all be used simultaneously; interpolation errors in the 180-deg interval and in the 90-deg interval then add at some orientations and cancel at others.

A second consistent difference between touch and vision is that the constant errors of touch interpolation are larger than the visual. The touch constant errors seem to be about twice as large as the visual errors, in general, with maximum average displacements of $4 \%$ of the interval being common for vision, compared with $8 \%$ for touch. This difference could be accounted for by the fact that distances perceived by touch increase more rapidly as a function of physical distance than do visually perceived distances.

\section{REFERENCES}

BARTLETT, N. R., REED, J. D., \& DUVOISIN, G. Estimation of distance on polar coordinate plots as a function of the scale used. Journal of General Psychology, 1949, 41, 47-65.

BORING, E. G. The sensations of the alimentary canal. A merican Journal of Psychology, 1915, 26, 1-57.

CARR, W. J., \& GARNER, W. R. The maximum precision of reading fine scales. Journal of Psychology, 1952, 34, 85-94.

CHAPANIS, A., \& LEYZOREK, M. Accuracy of visual interpolation between scale markers as a function of the number assigned to the scale interval. Journal of Experimental Psychology, 1950, 40, 655-667.

CHARLES, J. P., \& DUNCAN, C. P. The distance gradient in kinaesthetic figural aftereffects. Journal of Experimental Psychology, 1959, 57, 164-170.

CHENG, M. F. Tactile-kinaesthetic perception of length. American Journal of Psychology, 1968, 81, 74-82.

CHURCHILL, A. V. A comparison of tactual and visual interpolation. Canadian Journal of Psy chology, 1959, 13, 23-27.

CHURCHILL, A. V. Tactual and visual interpolation: A cross-modal comparison. Canadian Journal of Psychology, 1960, 14, 183-190.

GIBSON, J. J. Observations on active touch. Psychological Review, 1962, $69,477-491$.

HENRI, V. Recherches sur la localisation des sensations tactiles. Archives de Physiologie, 1893, Ser. 5, 5.

HENRI, V. Recherches sur la localisation des sensations tactiles. Année Psychologie, 1895, 3, 168-192.

JASTROW, J. On the judgment of angles. American Journal of Psychology, $1893,5,214-248$

KATORI, H., \& NATORI, K. The immediate reproduction of tactually perceived figures and the drawing process in reproducing the figures. Japanese Journal of Psychology, 1967, 38, 121-136 (in Japanese).

PILLSBURY, W. B. Some questions of the cutaneous sensibility. American Journal of Psy chology, 1894, 7, 42-57.

STFVFNS, S. S., \& GUIRAO, M. Subjective scaling of length and area and the matching of length to loudness and brightness. Journal of Experimental Psychology, 1963, 66, 177-186.

TAYLOR, M. M. Fffect of anchoring and distance perception on the reproduction of forms. Perceptual \& Motor Skills, 1961, 12, 203-230.

TAYLOR, M. M. Figural after-effects: A psychophysical theory of the displacement effect. Canadian Journal of Psychology, 1962, 16, 247-277

WONG, R. Fffect of the modulus on estimates of magnitude of linear extent. American Journal of Psychology, 1963, 76, 511-512.

\section{NOTES}

1. Present address: Department of Psychology, Lniversity of Wisconsin Madison, Wisconsin 53706 .

2. Address: Defence Research Establishment Toronto, Box 2000, Downsview, Ontario, Canada.

(Accepted for publication February 14, 1969.) 